with decreased LOC whose family asks for an ACP discussion in the ED. Participants were divided into groups (5-6 members). Each small group analyzed and discussed each case before the participants reconvened and discussed their opinions in one large group. ACP experts from palliative care, emergency medical services and EM facilitated the discussions highlighting the best practices from the literature for each case reviewed. Pre and post Likert surveys were distributed to workshop participants to assess changes in confidence in a variety of domains. A Wilcoxon Signed Rank Test showed statistically significant improvement in learner confidence within the following areas $(\mathrm{N}=21$; $\mathrm{p}<0.05$ ): identifying patients appropriate for GOC discussions, initiating GOC discussions, and identifying barriers to GOC, in the ED. The majority $(89 \%)$ of participants agreed the workshops should become part of our academic curriculum. Conclusion: An ACP/GOC workshop was successfully implemented and further ACP/GOC sessions are planned for the upcoming academic year. Looking ahead, we will look at using other teaching modalities such as simulation to further enhance the delivery of the curriculum. We will also attempt to capture defined physician behaviors (e.g. documenting GOC in the ED chart, sending letters to family physicians documenting GOC discussions) to gauge uptake of the workshop principles into clinical practice.

Keywords: innovations in emergency medicine education, advanced care planning, goals of care

\section{MP19 \\ Interprofessional airway microskill checklists facilitate the deliberate practice of direct intubation with a bougie and airway manikins}

J. P. French, MB, BSc, K. David, BN, S. Benjamin, BN, J. Fraser, BN, J. Mekwan, MBBS (Lond), P. Atkinson, MB, BCh, BAO, MA, Dalhousie University, Rothesay, NB

Introduction: Deliberate practice (DP) is the evolution of practice using continually challenging and focused practice on a particular task. DP involves immediate feedback, time for problem-solving and evaluation, and opportunities for repeated performance. Mircroskills training breaks down larger tasks into multiple smaller subtasks and then adds opportunities for feedback and adjustment for each subtask. Microskills training is routinely used to achieve excellence in competitive sports, martial arts, military operations, and music. Endotracheal intubation is a complex task with a clinically significant complication and failure rate. Methods: Two doctors and three nurses developed stepwise team microskills checklist from case review, simulations and published evidence. The checklist was tested, evaluated and developed during four days of simulation faculty team training. The final 36 item checklist was used to facilitate skills training for doctors, nurses, respiratory therapists and ACPs in one level 2, and two level 3 trauma centers from April 2017 to October 2017. The microskills checklist was used in four phases: 1. Group discussion of each microskill step 2. Groups of three team members; operator, assistant and microskill facilitator (using the checklist) to enable the deliberate analysis of the teams current performance. Each subtask is performed with immediate peer and where necessary faculty feedback. Changes are recorded. 3. Total task run though without interruption. Changes are recorded. 4. Repetition and feedback using different team members, manikins, including time pressure. User satisfaction surveys were collected after the skills training session Results: Results. Teams were composed of Registered Nurses (8), Physicians (9), and Respiratory Therapists (2). All of the teams experienced a change in practice. The median number of microskills changed for MDs 13/30, RNs 7/16. The commonest changes in practice were patient positioning (all teams). All professions agreed strongly that the approach produces a positive change in practice (median score 4.8/5). Conclusion: Microskills checklist facilitate endotracheal intubation with a bougie skill development in interprofessional teams in this provisional analysis.

Keywords: innovations in emergency medicine education, airway management, deliberate practice

\section{MP20}

ImageSim - performance-based medical image interpretation learning system

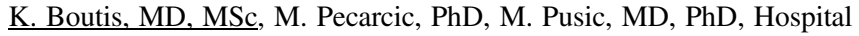
for Sick Children and University of Toronto, Toronto, ON

Introduction: Medical images (e.g. radiographs) are the most commonly ordered tests in emergency medicine. As such, emergency medicine physicians are faced with the task of learning the skill of interpreting these images to an expert performance level by the time they provide opinions that guide patient management decisions. However, discordant interpretations of these images between emergency physicians and expert counterparts (e.g. radiologists) is a common cause of medical error. In pediatrics, this problem is even greater due to the changing physiology with age. Methods: ImageSim (https://imagesim. com) is an evidence-based on-line learning platform derived and validated over an 11 year period (https://imagesim.com/research-andefficacy). This learning system incorporates the concepts of cognitive simulation, gamification, deliberate practice, and performance-based competency in the presentation and interpretation of medical images. Specifically, ImageSim presents images as they are experienced in clinical practice and incorporates a normal to abnormal ratio is representative of that seen in emergency medicine. Further, it forces the participant to commit to the case being normal or abnormal and if abnormal, the participant has to visually locate the specific area of pathology on the image. The participant submits a response and gets text and visual feedback with every case. After each case, the participant gets to play again until they reach a desired competency threshold $(80 \%$ is bronze resident; $90 \%$ silver staff emergency medicine physician; $97 \%$ gold radiologist). Importantly, the learning experience also emphasizes deliberate practice such that the learning system provides hundreds of case examples and therefore each participants performance has the opportunity to improve along their individual learning curve. Results: Course selection was made based on known medical image interpretation knowledge gaps for practicing emergency physicians. Currently, ImageSim live courses include pediatric musculoskeletal radiographs $(2,100$ cases, 7 modules) and pediatric chest radiographs (434 cases). In 2018, we will also release a pediatric point-of-care ultrasound course (400 cases, 4 modules) and the pre-pubertal female genital examination (150 cases). For a demo, go to https://imagesim.com/demo. Using ImageSim, the deliberate practice of about 120 cases ( 1 hour time commitment) increases accuracy on average by $15 \%$. Currently integrated into 10 emergency medicine training programs and there are about 300 continuing medical education world-wide participants. Conclusion: While acquiring mastery for these images may take years to acquire via clinical practice alone, this learning system can potentially help achieve this in just a few hours.

Keywords: deliberate practice, computer assisted learning, competency

\section{MP21}

Global emergency medicine fellowship: establishing a global EM training program at Queen's University

A. Collier, MD, S. A. Bartels, MD, MPH, D. Messenger, MD, Department of Emergency Medicine, Queen's University, Kingston, ON 